\title{
Curcumin nanoparticles and blue laser irradiation in photothermal inactivation of selected oral pathogens in vitro
}

\author{
Pavel Polenik \\ Charles University, Medical Faculty \\ Alej Svobody 80, Czech Republic \\ polenik@fnplzen.cz
}

\begin{abstract}
In last years, devices emitting laser beam in blue spectrum has been the subject of substantial research also in dentistry. But improvement of bactericidal effect might be theoretically possible using exogenous chromophore under the terms of photothermal therapy. With respect to the colour, curcumin seems be a suitable chromophore for this method and we decided to use curcumin nanoparticles and evaluate their effect as a photosensitizer for $445 \mathrm{~nm}$ laser in effectiveness against selected $\mathrm{G}^{+}$and $\mathrm{G}^{-}$bacteria in vitro. Curcumin nanoparticles were prepared using ultrasound assisted solvent-antisolvent precipitation. Diode laser $445 \mathrm{~nm}$ (SIROLaser Blue) was used as a source of laser irradiation $\left(100 \mathrm{~mW}, 1 \mathrm{~min}\right.$. exposition time, energy density $\left.7,68 \mathrm{~J} / \mathrm{cm}^{2}\right)$. Bacterial strains of Porphyromonas gingivalis, Parvimonas micra and Enterococcus faecalis were cultivated on solid media in marked areas covered by curcumin nanoparticles. After laser irradiation and subsequent anaerobic cultivation, the bacterial growth was evaluated. Irradiated areas without contact with curcumin nanoparticles showed intact bacterial colonies. Completely different quality was detectable in cases of irradiated colonies growing in contact with curcumin nanoparticles. Destruction of bacterial colonies was clearly visible and repeated cultivation of taken material was without positive response confirming presence of non-living cells inside of colonies. Results of our preliminary study showed promising direction of laser photothermal therapy using curcumin nanoparticles in reduction or elimination of oral microflora especially in locations with poor access for classic therapeutic effect.
\end{abstract}

Keywords: photothermal effect, curcumin, nanoparticles, blue laser

\section{Introduction}

More than 750 types of bacteria or phylotypes are detected in oral cavity. Despite the fact, that human immune system constantly monitores their growth and reproduction, some of the bacteria in the mouth are responsible for oral diseases such as caries and periodontal diseases, which are one of the most common diseases in humans. Prevention and treatment are consequently focused on reduction of bacterial load of oral structures. According to the location of bacterial biofilm different methods of antibacterial strategy can be used. Serious problem is accessibility of bacterial communities localized in deep periodontal pockets and, also between and inside of epithelial cells. Systemic application of antibiotic is frequently the method of choice in these cases. But the undesirable side effect of such therapeutic access is general decrease of sensitivity to antibiotics. So that the continual looking for an alternative method is the imperative of present time.

Light from high-power lasers is known to be bactericidal and investigations have shown that it is effective against organisms implicated in caries and inflammatory periodontal diseases. Although light from low-power lasers has no adverse effect on bacterial viability, bacteria can be sensitized to killing by such light by prior treatment with a chemical photosensitizing agent. The advantages of the technique are that killing is achieved in very short periods of time $(<60 \mathrm{~s})$, resistance development in the target bacteria would be unlikely and damage to adjacent host tissues can be avoided. This approach may be a useful alternative to antibiotics and antiseptics in eliminating cariogenic and periodontopathogenic bacteria from disease lesions.

Moreover, laser delivery systems have more better access to the locations where the effectivity of classic mechanical methods of biofilm removal is questionable. Different wavelengths in combinations with responsible photosensitizers have been tested in connection with photodynamic (PDT) inactivation of selected oral pathogens. As PDT is an oxygendependent process, its use in deep periodontal pockets and infected root canals may be limited. From this point of view, killing of microorganisms through a local hyperthermal mechanism - antimicrobial photothermal therapy (PTT) might by more effective. 
In last years, devices emitting laser beam in blue spectrum has been the subject of substantial research also in dentistry. Previous investigations demonstrated phototoxic effect of blue light for effectively reducing some pathogens. But improvement of bactericidal effect might be theoretically possible using exogenous chromophore under the terms of PTT. With respect to the colour, curcumin seems be a suitable chromophore for this method. In addition to this activity, antiinflammatory, antioxidant, chemopreventive and chemotherapeutic abilities may be beneficial in suppression of inflammatory reactions.

Important criterion of effectivity of photothermal reaction is accessibility and contact of bacteria with photosensitizer. Because curcumin shows poor solubility in water, we decided to use curcumin nanoparticles and evaluate their effect as a photosensitizer for $445 \mathrm{~nm}$ laser in effectiveness against selected $\mathrm{G}^{+}$and $\mathrm{G}^{-}$bacteria in vitro.

\section{Materials and methods}

Curcumin nanoparticles preparation

Stock of curcumin solution $(5 \mathrm{mg} / \mathrm{ml}$ ) was prepared by dissolving curcumin powder (Sigma) in $90 \%$ ethanol (sample E) or acetone (sample A) $(20 \mathrm{ml})$. One $\mathrm{ml}$ of stock solution was added to boiling water $(50 \mathrm{ml})$ in drop-wise manner under ultrasonication condition with an ultrasonic power and frequency of $50 \mathrm{kHz}$. The solution was sonicated for $30 \mathrm{~min}$. After sonication, the mixture was stirred at $800 \mathrm{rpm}$ for $20 \mathrm{~min}$ till the orange coloured precipitate was obtained. Thereafter, supernatant was discarded and the pellet obtained was used for further study. Before application on the surface of solid cultivation media $100 \mathrm{mg}$ of curcumin nanomaterial was dissolved in $1 \mathrm{ml}$ of $0,9 \%$ saline solution.

Laser equipment

Diode laser emitting $445 \mathrm{~nm}$ laser beam (SIROLaser Blue, Dentsply Sirona, Germany) was used for evaluation of spontaneous antibacterial laser effect and for induction of photothermal reaction in cooperation with curcumin nanoparticles.

Bacterial strains of Porphyromonas gingivalis ATCC 33277, Parvimonas micra ATCC 33270 and Enterococcus faecalis ATCC 29212 were obtained from Thermo-Scientific (Lenexa, KS, USA). All the three strains were cultivated into anaerobe basal broth (Oxoid, Basingstoke, Hampshire, England) under anaerobic conditions for 48 hours at $37^{\circ} \mathrm{C}$. Then $P$. gingivalis and $P$. micra were inoculated on anaerobe basal agar (Oxoid, Basingstoke, Hampshire, England) supplemented with 5\% sterile defibrinated horse blood. Rounded contact areas of $10 \mathrm{~mm}$ diameter were formed [Fig.1] and followed cultivation under anaerobic conditions to the mid-log phase at $37^{\circ} \mathrm{C}$. E. faecalis was inoculated on Slanetz\&Bartley medium and the same process of preparation and cultivation was done. 


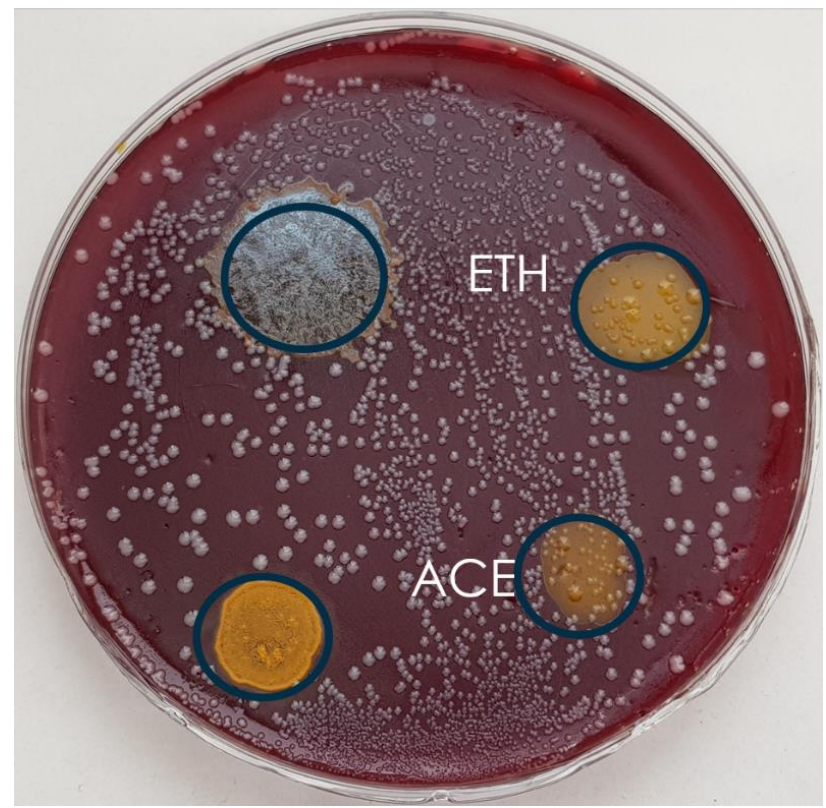

Fig.1 Colonies of $P$. micra covering curcumin nanoparticles.

ETH- solvent=ethanol, ACE- solvent=acetone.

Subsequently, both areas containing curcumin nanoparticles and one colonized area free of nanoparticles of the same extent were irradiated by the laser. Following settings were used: $100 \mathrm{~mW}$ power, irradiation time $1 \mathrm{~min}$, fiber $0,3 \mathrm{~mm}$, distance of fiber from the surface $15 \mathrm{~mm}$, energy density $7,68 \mathrm{~J} / \mathrm{cm}^{2}$ [Fig.2]. Complete test for each of observed bacteria was 10 times repeated.

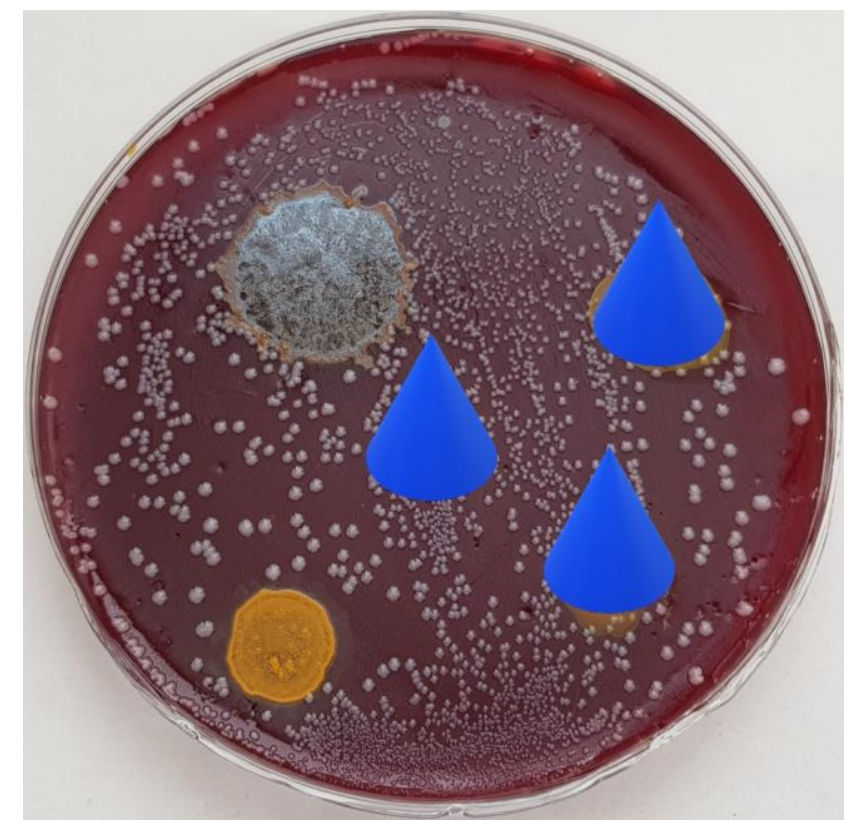

Fig.2 Irradiation of areas containing curcumin nanoparticles from Fig. 1 and without nanoparticles [left area]. After 72 hours of anaerobic cultivation the antibacterial effect was evaluated. 


\section{Results}

The same uniform picture was visible on the surface of 30 bacterial cultures. Irradiated areas without contact with curcumin nanoparticles showed intact bacterial colonies. Subsequent cultivation samples from these colonies validated viability of colony forming bacteria. Completely different quality was detectable in cases of irradiated colonies growing in contact with curcumin nanoparticles. Destruction of bacterial colonies was clearly visible and repeated cultivation of taken material was without positive response confirming presence of nonliving cells inside of colonies. The same bactericidal effect was observed in both used solvents (ethanol, acetone) [Fig.3].

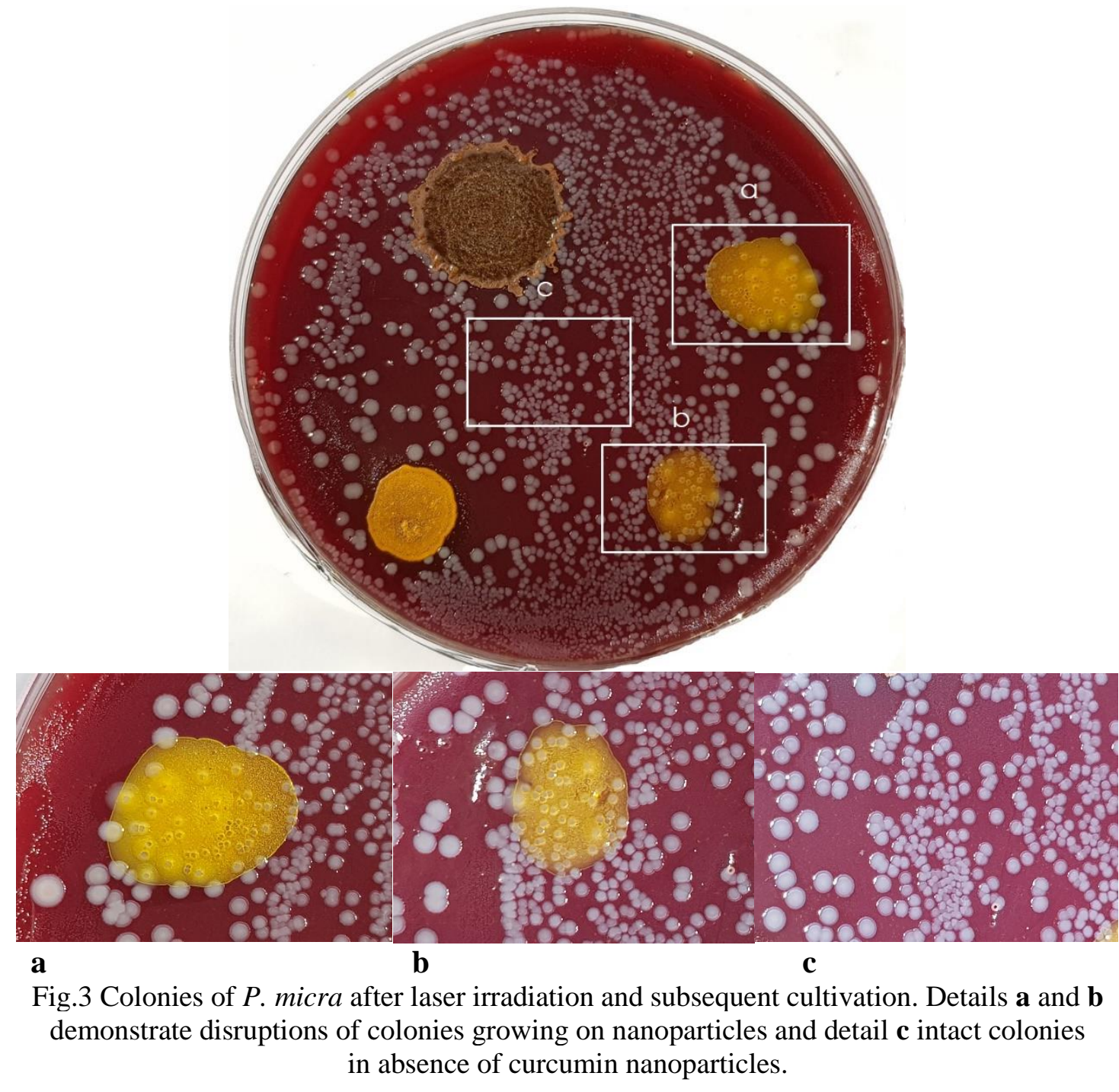

\section{Discussion}

The goal of antimicrobial therapy in dentistry is simple in principle yet often difficult to achieve: to eradicate periodontal pathogens in the oral cavity. The difficulty arises not from the antimicrobial agents themselves but from the complex array of reservoirs where these organisms can persist and means to effectively deliver antimicrobial agents to these locations. Important questions of antibacterial strategy are also different forms of bacterial colonization associated 
with dissimilar sensitivity to the antimicrobials. Especially in periodontology, the dominant problem is complexity of bacterial biofilm. It is possible to found in recent bibliography a rich list of substances with antibacterial effect, but the methods of evaluation are not usable for practical use. It is mostly detection of bactericidal activity in planktonic cultures, but in reality, the dominant problem is very resistant bacterial biofilm. In planktonic cultures curcumin solutions inhibited the growth of $P$. gingivalis in dose-dependent manner and its MIC was $12 \mu \mathrm{g} / \mathrm{ml}$ [1]. On the other side, concentration of 20 $\mu \mathrm{g} / \mathrm{ml}$ was able to inhibit P. gingivalis biofilm formation by $>80 \%$ [2]. Next step in the use of curcumin is connected with laser application in the terms of photodynamic therapy. In the context of combination of curcumin solutions and laser energy the reduction of salivary bacteria is better than curcumin or diode laser alone [3]. To a lesser extent was the research focused on evaluation of single-species [4] and multi-species biofilms [5]. On this field of activity is dominant problem accessibility of target structures and significant differences are typical for such antibacterial activities, Nanotechnology strategies for curcumin delivery have been studied over the past years. A diverse array of novel preparations has been developed (nano-curcumins) and these nano-range formulations have been mostly limited to in vitro models of cancer. But some investigations have shown that the formulations have also potential for the treatment of infection diseases.

\section{Conclusion}

Curcumin nanoparticles showed in our study very good penetration into bacterial colonies forming suitable location of chromophore for corresponding laser beam. The results demonstrate simultaneously promising direction in reduction or elimination of oral microflora especially in locations with poor access for classic therapeutic methods.

\section{References}

[1] A. M. Sha, B. T. Garib, „Antibacterial effect of curcumin against clinically isolated Porphyromonas gingivalis and connective tissue reactions to curcumin gel in the subcutaneous tissue of rats, “ BioMed Res. Int., vol.2019, article 6810936, 14 pages.

[2] S. Izui, S. Sekine, K. Maeda, M. Kubowina, A. Takada, A. Amano, H. Nagata, „Curcumin inhibited the growth of P. gingivalis in dose-dependent manner,“ J. Periodontol., vol. 87, no.1, pp. 83-90, 2016.

[3] D. P. Leite, F. R., Paolillo, T. N. Parmesano, C. R. Fontana, V. S. Bagnato, „Effects of photodynamic therapy with blue light and curcumin as mouth rinse for oral disinfection: a randomized controlled trial." Photomed. Laser Surg., vol. 32, no. 11, pp. 627-632, 2014.

[4] M. C. Andrade, A. P. D. Ribeiro, L. N. Dovigo, I. L. Brunetti, E. T., Giampaolo, „Effect of different pre-irradiation times on curcumin mediated photodynamic therapy against planktonic cultures and biofilm of Candida spp.," Arch. Oral Biol., vol. 58, no. 2, pp. 200-210, 2013.

[5] N. C. Zaranjo, C. R. Fontana, V. S. Bagnato, M. E. M. Gerbi, „, Photodynamic antibacterial therapy of curcumin in biofilms and carious dentine,“ Lasers Med. Sci., vol. 29, no. 2., pp. 629-635, 2014. 